\title{
A Case Report on Linezolid and Cefuroxime Induced Leucocytoclastic Vasculitis
}

\author{
Aparna R Menon ${ }^{1}$, Bhanu Kumar ${ }^{2}$, Koneru Vasavi ${ }^{1}$, Sulfath TS ${ }^{1}$, Sri Harsha Chalasani ${ }^{1, *}$, Madhan Ramesh ${ }^{1}$ \\ 'Department of Pharmacy practice, JSS College of Pharmacy, Mysuru, Jagadguru Shri Shivarathreeshwara Academy of Higher Education and Reasearch, SS Nagar, \\ Mysuru, Karnataka, INDIA. \\ ²Department of General Medicine, JSS Medical college and Hospital, JSS Academy of Higher Education and Research, Mysuru, Karnataka, INDIA.
}

\begin{abstract}
A 65-year-old female diabetic patient with wet gangrene on left great toe developed skin eruptions and blebs over her limbs and lower abdomen after receiving three doses of tablet linezolid + cefuroxime $1600 \mathrm{mg}+500$ $\mathrm{mg})$. The skin eruptions and blebs completely resolved fourteen days after discontinuation of tablet Linezolid + Cefuroxime. Based on her presentation (Skin eruptions and blebs), we consider that the condition was a result of linezolid+ cefuroxime administration. While the pathophysiology of these cutaneous reactions is not completely understood, clinicians should be vigilant to allow early detection of these problems. The causality of this adverse reaction was determined by using Naranjo's criteria and World Health Organization Probability scale and was found to be possible and the severity of this reaction was determined by using the Modified Hartwig and Siegel scale and was found to be moderate (Level $3 b$ ) reaction. Although, Linezolid is known to cause dermatological reactions like rashes
\end{abstract}

but the reports of leucocytoclastic vasculitis are rare. Thus, our case report of linezolid + cefuroxime induced leucocytoclastic vasculitis add newer information. Physician should be vigilant for the potential of drug to cause some rare side-effect like leucocytoclastic vasculitis on similar age groups, so that a safer alternative treatment can be started.

Key words: Linezolid, Cefuroxime, Cutaneous reaction, Diabetes, Wet gangrene.

Correspondence

Dr. Sri Harsha Chalasani, Faculty, Department of Pharmacy Practice, JSS College of Pharmacy, Mysuru-570015, Karnataka, INDIA.

Email: sriharshachalasani@jssuni.edu.in

DOI: 10.5530/jyp.2019.11.47

\section{INTRODUCTION}

Linezolid is a synthetic antibiotic, first of the oxazolidinone class, used for the treatment of infections caused by gram positive, certain gram negative, anaerobic bacteria and multi-resistant bacteria including streptococcus and Methicillin-resistant Staphylococcus Aureus (MRSA). The drug works by inhibiting the initiation of bacterial protein synthesis by binding to the $50 \mathrm{~S}$ subunit of the prokaryotic ribosome, preventing formation of the initiation complex for protein synthesis. ${ }^{1,2}$ Adverse effects associated with linezolid range from nausea, vomiting to severe myelosuppression while cutaneous reactions are rare. While, Cefuroxime belongs to the second-generation semi-synthetic cephalosporin and indicated in the treatment of bronchitis ${ }^{1,3}$ The common reactions to cephalosporins include a maculopapular or morbilliform skin eruption, drug fever and a positive antiglobulin or coombs test. Less common reactions may include urticaria, eosinophilia, serum-sickness-like reactions and anaphylaxis. ${ }^{4,5}$ We report a case of Leucocytoclastic Vasculitis (LCV) in a geriatric female patient who had neither previous history of drug allergies nor cutaneous reactions and was presumably caused by linezolid+cefuroxime. Leukocytoclastic vasculitis, also known as hypersensitivity vasculitis and hypersensitivity angitis, is a histopathologic term commonly used to denote a small-vessel vasculitis. Histologically, LCV is characterized by leukocytoclasis, which refers to vascular damage caused by nuclear debris from infiltrating neutrophils. ${ }^{6}$

\section{CASE NARRATION}

A 65-year-old female patient was admitted to a tertiary care hospital with complaints of reddish lesions over both the lower limb and lower half of abdomen. Patient was explained about the drug reaction and her written consent was sought on an appropriate informed consent form. Figure $1,2$.

Initially she was admitted to a local Hospital due to a wound over her left great toe, for which wound debridement was done and patient was prescribed with tablet linezolid+ cefuroxime $(600 \mathrm{mg}+500 \mathrm{mg})$. After receiving three doses of Linezolid + Cefuroxime she developed multiple drug induced eruptions and blebs from lower half of the abdomen and both lower limbs. The patient's past medical history was suggestive of Type II diabetes mellitus since 11 years and she was on regular treatment. On examination, her heart rate was $92 \mathrm{bpm}$, blood pressure was 130/80 $\mathrm{mmHg}$ and respiratory rate was 22 cycles per minute. Other systemic investigations were clinically unremarkable. Based on her complaints, a clinical diagnosis of type 2 diabetes mellitus with wet gangrene of left great toe with drug induced small vessel vasculitis was provisionally made. Relevant investigations to evaluate her diabetes control and gangrene were advised. Her random glucose level were $383 \mathrm{mg} / \mathrm{dl}$ and Erythrocyte Sedimentation Rate (ESR) was $130 \mathrm{~mm} / \mathrm{hr}$. Her left lower limb arterial doppler was done and the impression was generalized atherosclerotic peripheral vascular disease with thrombosis of the distal $2 / 3^{\text {rd }}$ of Posterior Tibial Artery (PTA). Following the hospitalisation, patient was treated with Inj. H actrapid 20U TID, T. Paracetamol 650mg, Inj. Pantoprazole 40mg and Inj. Tinidazole 400mg. Further, T. Linezolid+ Cefuroxime was discontinued along with dermatologist and clinical pharmacist opinion which was sought for the management of drug induced vasculitis. Inj. Meropenam $1 \mathrm{~g}$ was started as an alternative treatment for wet gangrene. 


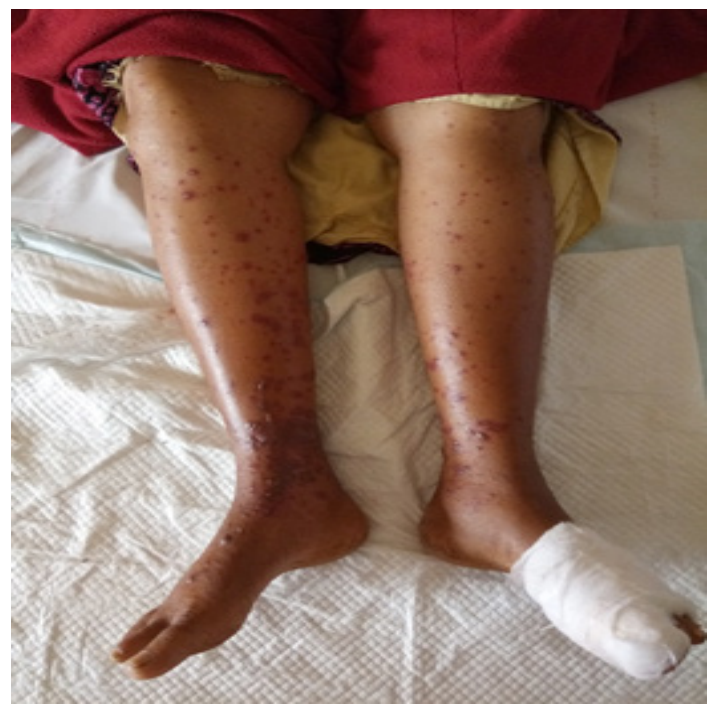

Figure 1: Reaction Developed on Legs of Patient.

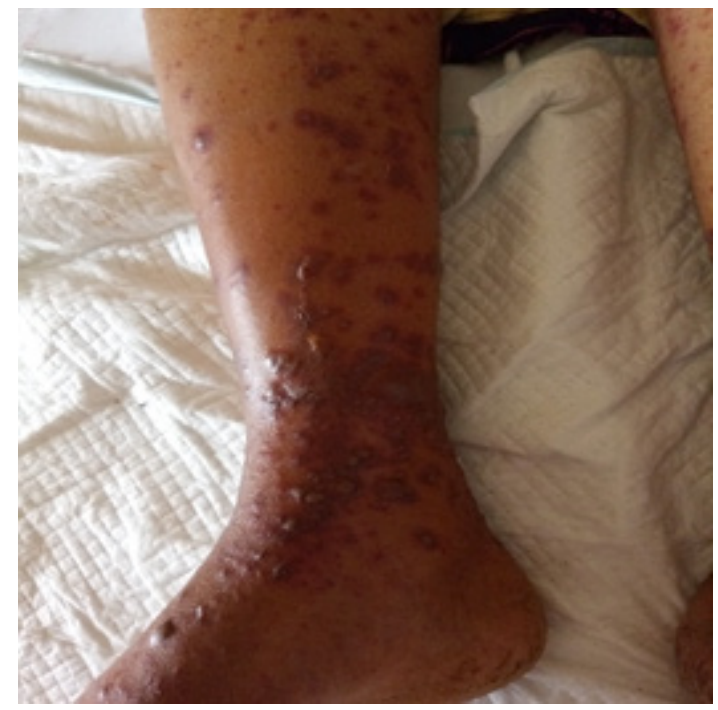

Figure 2: Reaction Developed on Legs of Patient.

Clinical pharmacist and Dermatologist together opines this reaction as drug induced leucocytoclastic vasculitis secondary to linezolid+ cefuroxime. The patient was symptomatically treated with fusidic acid cream $20 \mathrm{mg}$ and ketoconazole cream $2 \%$ twice daily. Biopsy reports confirmed leucocytoclastic vasculitis. Following the above said treatment, although the patient reported a mild relief from the symptoms, the skin eruptions were persistent. However, after two days of discontinuation of suspected drugs the lesions started to subside and on $14^{\text {th }}$ day the reaction disappeared leaving only fewer scars on skin. Figure 3.

During discharge, the remaining drugs, Inj. H actrapid 20U-20U-20U, Tab. Methylcobalamine, Thrombophobe ointment (Benzyl Nicotinate $2 \mathrm{mg}+$ Heparin Topical 50 IU) was continued.

The Naranjo's criteria and World Health Organization probability scale were applied to determine the causality for the suspected Adverse Drug Reaction (ADR). The causality assessment with both scales revealed that the ADR was due to linezolid+cefuroxime in this patient was "possible". The severity of the ADR was determined by using the Modified Hartwig and Siegel scale and was found to be moderate (Level 3b) reaction.

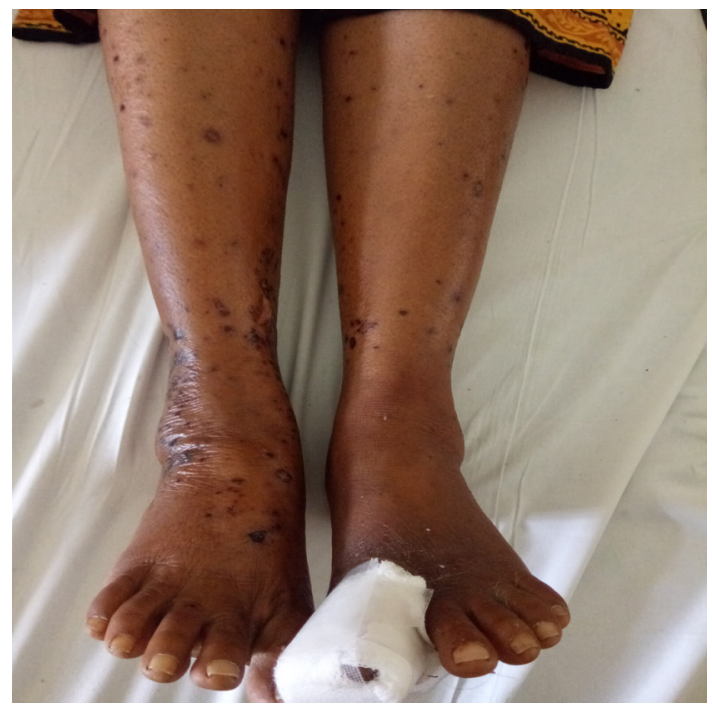

Figure 3: Subsiding of Reaction on Legs of Patient.

\section{DISCUSSION}

Linezolid is an oxazolidinone and acetamide derived anti-bacterial agent and functions as protein synthesis inhibitor that is used in the treatment of gram-positive bacterial infections (Complicated and uncomplicated skin and skin structure infections, methicillin resistant staphylococcal infections) and respiratory tract (Community acquired pneumonia, nosocomial pneumonia). The major adverse effect associated with linezolid include lactic acidosis, peripheral neuropathy, optic neuropathy, myelosuppression, seizure and hepatic injury. Cutaneous reactions associated with linezolid is very rare. Pathogenesis for linezolid induced leucocytoclastic vasculitis is not completely understood.

Leukocytoclastic Vasculitis (LCV), also known as hypersensitivity vasculitis and hypersensitivity angilitis, is a histopathologic term commonly used to denote a small-vessel vasculitis. LCV is characterized by leukocytoclasis, which refers to vascular damage caused by nuclear debris from infiltrating neutrophils. ${ }^{6} \mathrm{LCV}$ may be secondary to medications, underlying infection, collagen-vascular disorders, or malignancy. The suspected risk factors that contribute to the aggravation of the $\mathrm{cu}$ taneous reaction are gender of the patient. ${ }^{6}$ In this case, the cutaneous reaction was observed after receiving three doses of tablet linezolid + cefuroxime. The reaction developed initially over her both lower limbs which later on aggravated to lower abdomen. Vasculitis is very rare adverse event of linezolid. Until now, to the best of our knowledge, only one case has been reported in Journal of Scientific Society. ${ }^{1}$ However, till date there are no case reports suggesting cefuroxime causing leucocytoclastic vasculitis. Thus, in this patient, the temporal relationship between the linezolid+ cefuroxime uses and the onset of cutaneous reaction and its resolution when the drug was stopped is plausible and strongly indicates linezolid+ cefuroxime are responsible for the suspected reaction.

\section{CONCLUSION}

Although, Linezolid is known to cause dermatological reactions like rashes but the reports of leucocytoclastic vasculitis are rare. Thus, our case report of linezolid + cefuroxime induced leucocytoclastic vasculitis add newer information. Physician should be vigilant for the potential of drug to cause some rare side-effect like LCV on similar age groups, so that a safer alternative treatment can be started. The patients should be identified and promptly reported if any adverse drug reactions occur. 


\section{CONFLICT OF INTEREST}

The authors report no conflict of interest that is directly relevant to the content of the case report.

\section{REFERENCES}

1. Sathyanarayana V, Das U, Babu KG, Suresh TM, Babu S, Lakshmaiah KC. Linezolid induced vasculitis: An unusual case report with review of the literature. J Sci Soc. 2015;42(1):27.
2. Kim FS, Kelley W, Resh B, Goldenberg G. Linezolid induced purpuric medication reaction. JCP. 2009;36(7):793-5.

3. Vardakas KZ, Ntziora F, Falagas ME. Linezolid: effectiveness and safety for approved and off-label indications. Expert Opinion on Pharmacotherapy. 2007;8(14):2381-400.

4. Sahoo HB, Rath B, Behera JP, Moharana CS. Cefuroxime axetil associated oral eruption: A case report. Apollo Medicine. 2016;13(2):133-4.

5. Saeed SA, Bazza M, Zaman M, Ryatt KS. Cefuroxime induced lymphomatoid hypersensitivity reaction. PMJ. 2000;76(899):577-9.

6. Daniel CL. Leukocytoclasticvasculitis and Henoch- Schönleinpurpura. Arthritis and Allied Conditions. 2005;86:1793-7.

Article History: Submission Date : 16-11-2018; Revised Date : 02-01-2019; Acceptance Date : 17-02-2019.

Cite this article: Menon AR, Kumar B, Vasavi K, Sulfath TS, Chalasani SH, Ramesh M. A Case Report on Linezolid and Cefuroxime Induced Leucocytoclastic

Vasculitis. J Young Pharm. 2019;11(2):227-9. 